\title{
Anabases
}

ANABASES Traditions et réceptions de l'Antiquité

$24 \mid 2016$

Varia

\section{Roger D. Woodard, The textualization of the Greek alphabet}

Mathilde Cambron-Goulet

\section{OpenEdition}

1 Journals

Édition électronique

URL : http://journals.openedition.org/anabases/5795

DOI : 10.4000/anabases.5795

ISSN : 2256-9421

Éditeur

E.R.A.S.M.E.

Édition imprimée

Date de publication : 10 novembre 2016

Pagination : 366-367

ISSN : 1774-4296

\section{Référence électronique}

Mathilde Cambron-Goulet, "Roger D. Woodard, The textualization of the Greek alphabet », Anabases [En ligne], 24 | 2016, mis en ligne le 15 novembre 2016, consulté le 23 septembre 2020. URL : http:// journals.openedition.org/anabases/5795 ; DOI : https://doi.org/10.4000/anabases.5795

Ce document a été généré automatiquement le 23 septembre 2020.

(c) Anabases 


\title{
Roger D. Woodard, The textualization of the Greek alphabet
}

\author{
Mathilde Cambron-Goulet
}

\section{RÉFÉRENCE}

Roger D. Woodard, The textualization of the Greek alphabet, Cambridge / New York, Cambridge University Press, 2014, $367 \mathrm{p}$.

74, 99 livres / isbn 9781107028111

1 Woodard propose une double étude dans laquelle le geste d'écriture comme performance est examiné à partir de plaques de cuivre sur lesquelles sont gravés des abécédaires. Les deux études, quoique inter-reliées, peuvent être lues séparément, mais la profondeur de l'ouvrage repose sur leur juxtaposition.

2 La première moitié de l'ouvrage porte sur des plaques de cuivre, l'une de la collection de Würzburg (W) et deux de celle de M. Schøyen (MS), gravées d'abécédaires comportant les lettres de $\alpha$ à $\tau$. Après un chapitre sur l'histoire récente des plaques qui explique leur origine obscure, Woodard analyse systématiquement les différentes formes que prennent sur les plaques les vingt-deux lettres de l'alphabet. La comparaison avec d'autres documents (vases, inscriptions) permet de caractériser les différents glyphes employés pour chacune des lettres sur le plan spatio-temporel. Il s'agit d'un chapitre très technique, qui a le mérite d'être clair, même s'il n'est pas toujours suffisamment illustré pour qui n'a pas en tête les différents yod ou kappa qu'on trouve sur tel vase ou dans tel graffiti. L'ouvrage étant accompagné d'un petit site internet sur lequel se trouvent des illustrations des plaques, il eût été utile d'y inclure des images des principaux vases et des principales inscriptions citées. Le troisième chapitre, signé par D. A. Scott, propose une étude physique et chimique des plaques, suffisamment bien vulgarisée pour que le lecteur, même peu versé dans les méthodologies utilisées, puisse la comprendre. Son travail permet de montrer que les plaques sont authentiques; qu'elles sont liées entre elles en raison des composés qui 
leur donnent leur patine, lesquels montrent qu'elles sont issues d'une même feuille de cuivre ; enfin, que des résidus de bois et de matière organique y révèlent des traces d'inhumation. L'intérêt de ce chapitre tient à la provenance inconnue des plaques, qui ne peut être identifiée à l'aide de critères externes. Woodard reprend au chapitre 4 son étude sur les abécédaires, qu'il transcrit en signalant les « anomalies » : distorsions par rapport à l'ordre alphabétique habituel, séquences de lettres omises, etc. Deux transcriptions distinctes permettent dans un cas de distinguer les différents glyphes utilisés, et dans un autre cas facilitent la lecture à l'aide d'une transcription orthographique commune. Les anomalies les plus communes que révèlent les plaques sont les confusions entre $x i$ et êta et entre thêta et omicron, et l'omission de la séquence $\kappa \lambda \mu v \xi_{0}$. Les images disponibles en ligne sont très utiles pour suivre l'argumentation des chapitres 3 et 4 .

3 La seconde étude s'ouvre sur des considérations sur le caractère arbitraire des signes sonores et graphiques qui constituent la langue et l'écriture. Woodard rappelle que le nombre de graphèmes ne correspond pas nécessairement au nombre de phonèmes ; que les graphèmes sont appelés à varier ; que l'usage tolère un certain écart par rapport au «signe standard» et une certaine homographie, pour peu qu'une personne compétente linguistiquement soit capable de retracer le signe langagier qu'encode le graphème. Il remarque que l'abécédaire (à tout le moins le noyau a-t) n'a pas changé d'ordre depuis les abécédaires phéniciens, alors que l'ordre des lettres dans l'abécédaire n'a pas d'impact sur le plan fonctionnel: la structure syntagmatique de l'abécédaire est transmise par la tradition. La langue et l'écriture étant deux phénomènes distincts, les plaques de cuivre appartiennent ainsi au domaine de l'écriture et non de la langue.

4 En examinant les différents symboles employés pour encoder différents phonèmes, et qui ont été identifiés dans les chapitres 2 et 4 , Woodard découvre que les séquences comportant des "anomalies" peuvent être lues non comme des abécédaires mais comme des mots. Le chapitre 6 porte sur une séquence de la plaque MS 2-2, ligne 16, qui peut se lire, en graphèmes habituels, $\mu \eta \lambda \eta \sigma \varepsilon \lambda v \zeta \eta \alpha \beta \gamma \delta$. La discussion qui suit vise à déterminer le sens de cette séquence, à l'aide de la linguistique historique et de témoignages archéologiques et littéraires. Woodard établit ainsi deux rapprochements : 1) entre le texte de la plaque et la tradition littéraire sur le sanctuaire d'Héra à Samos, où l'on trouve un sculpteur nommé Smilis et un arbre sacré (lugos) à partir duquel des guirlandes sont tressées ; 2) entre les plaques gravées d'abécédaires et d'autres formes d'écriture associées à des performances dans des contextes rituels tels que l'oracle de Dodone où la demande de révélation et la révélation elle-même sont écrites. La séquence $\mu \eta \lambda \eta \sigma \varepsilon \lambda \nu \zeta \eta \alpha \beta \gamma \delta$ signifierait alors : « abécédaire, que le stylet te tisse ».

5 Tous ces éléments se mettent en place dans le dernier chapitre, lorsque Woodard attire l'attention du lecteur sur la confusion entre langage et écriture et sur la métaphore du

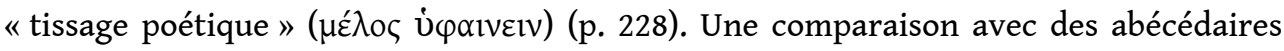
latins et hébreux permet de savoir que l'on avait l'habitude de réciter l'alphabet dans l'ordre, mais aussi en intercalant les éléments finaux entre les éléments initiaux ( $\alpha \omega$, $\beta \psi, \gamma \chi$, etc.) - ordre alphabétique «tissé » qui a pour effet d'attribuer à un graphème donné (placé à un certain endroit sur le fil de trame) le phonème encodé à la même position (fil de chaîne) dans l'abécédaire précédent ( $\omega=\beta, \beta=\gamma, \psi=\delta$, etc.). Le même phénomène se produit en intercalant les lettres finales à partir du milieu $(\alpha \lambda \beta \mu \gamma v)$. Ce «tissage » de l'alphabet explique certains glissements d'un symbole à un autre pour 
représenter un même phonème, par exemple thêta et omicron (p. 258-259), la forme des lettres ayant tendance à être contaminée par celle des lettres qui lui sont contiguës dans l'abécédaire "tissé », ou lorsqu'elles se trouvent sur le même "fil de chaîne » et correspondent au même phonème. Ces alphabets tissés filent la métaphore du « tissage poétique " qui désigne la composition orale et la performance, et expriment la structure du langage dans ses composantes paradigmatique (fils de chaîne) et syntagmatique (fils de trame). Ils sont en cela des offrandes appropriées pour des

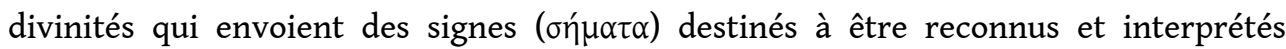

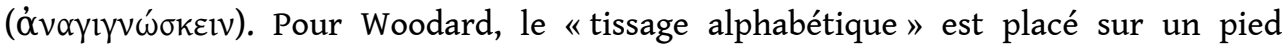
d'égalité avec le «tissage poétique » et signe par conséquent la démocratisation de la composition poétique : l'écriture permet à chacun de produire une performance, sans qu'il faille pour cela être un aède reconnu - même si ce n'est que par le tissage d'un abécédaire.

6 Woodard tisse avec cet ouvrage une toile complexe dans laquelle chaque fil finit par jouer un rôle. Lire les deux études séparément donne sans doute accès aux fils de chaîne et aux fils de trame, mais cela ne rendrait pas justice au travail de son artisan : une telle lecture, trop technique, ne permet pas de voir le tissu.

\section{AUTEURS}

\section{MATHILDE CAMBRON-GOULET}

Université du Québec à Montréal

cambron-goulet.mathilde@uqam.ca 